\title{
EMBODIED DIGITAL TWINS FOR ENVIRONMENTAL APPLICATIONS
}

\author{
Alexander Klippel, Pejman Sajjadi, Jiayan Zhao, Jan Oliver Wallgrün, Jiawei Huang, Mahda M. Bagher
}

Department of Geography/Center for Immersive Experiences, The Pennsylvania State University, 16802, University Park, PA, USA

KEY WORDS: Embodied cognition, immersive experiences, environmental modeling, place-based experiences.

\begin{abstract}
:
The synergies of advances in environmental sensing and modeling and the mainstreaming of immersive technologies lay the foundation for a theoretical grounding of embodied digital twins. Embodied digital twins draw on an established understanding of the importance of place for environmental sciences as well as a paradigmatic shift in the cognitive sciences toward embodied cognition. Nonetheless, the excitement of realizing embodied experiences through immersive technologies such as augmented and virtual reality stands in stark contrast to a lack of consistent terminology and empirical research. In this vision paper, we are proposing to draw more deeply on the theoretical basis of embodied cognition and to establish research frameworks for advancing embodied digital twins. We discuss several examples for turning environmentally sensed and modeled information into high-fidelity immersive experiences and provide a discussion and outlook on critical topics to address.
\end{abstract}

\section{INTRODUCTION}

Advances in computing and 3D modeling have nourished our capacity to create digital replicas of physical entities such as airplanes, production facilities, or forests. Digital Twins is a concept and term used predominantly in domains where the components of a system are well understood, for example, in manufacturing (Tao et al., 2019), allowing for detailed descriptions of data, components, behaviors, life cycles, and processes that are relevant to emulate the system's behavior, run simulations, and derive insights on effects of changes (Grieves and Vickers, 2017). For natural environments, other terms have been introduced to capture similar ideas and approaches, first and foremost virtual geographic environments (Lin et al., 2015); albeit, the complexity of the natural world poses additional challenges. With the increase of sensing, modeling, and predicting environmental conditions, it is time to think about opportunities and apply concepts developed and discussed in the context of digital twins to natural environments.

In parallel to developments in the realms of environmental sensing and modeling, we also witness the maturing of technologies for visualizing environmental data (Coltekin et al., 2020) and even more importantly, to make them experiential (Bailenson, 2018). Experiences are the foundation of human cognition and as such, play a key role in education, research, communication, and decision-making (Mandler, 1992). The emergence of mainstream immersive technologies, such as head-mounted displays (HMDs), allows for a paradigmatic shift in how environmental processes and changes are communicated and understood, how experts, lay people, and decision-makers ground their understanding not exclusively in data and traditional media but in embodied experiences enabled through augmented, mixed, and virtual reality, XR in short (Dede, 2009). XR technologies provide a platform for embodied experiences of data eradicating the dichotomy of traditional media that separate users from digital artifacts. But what do we know about the effect of embodying digital twins of environmental data?

Environmental and Earth Sciences are place-based disciplines that value, above all else, insights and discoveries that can best or exclusively be obtained through experiences in the field (Dolphin et al., 2019). Place, in environmental sciences, is equally important for education, research, communication, and decisionmaking. Discipline defining discoveries and observations are made in the field such as the theory of plate tectonics (Cox and Hart, 1986). There is also evidence that learning about environments is most effective in context, that is, in the field (Semken et al., 2018). Placing learners, researchers, and decision-makers into the real-world with a specific problem that is relevant to a location provides a more direct connection of key learning and discovery elements facilitating engagement and understanding (Powers, 2004). Places (the field), allow for discovery-based learning, which is founded in the constructivist approach where the act of discovery in problem solving is guided by a teacher or interactive systems through steps of scaffolded instruction (McComas, 2014). Embodied digital twins, as we will introduce them in this paper, allow for blending place-based and discovery-based learning to achieve "perceptual blending of the real and the virtual world with its place-based authenticity" to enable better learning and problem-solving experiences (Barab and Dede, 2007).

In this vision paper, we argue for embodied digital twins for environmental applications in research, education, communication and decision-making. We will discuss this concept against the backdrop of three examples of how advanced environmental sensing and modeling enables the creation of high-fidelity immersive virtual worlds (Section 3). While the implementation of the idea of embodied digital twins for environmental applications is made possible by the advancements of environmental sensing in combination with immersive technologies, and the recent years have brought forward a number of commercial endeavours in this area (Google Earth VR ${ }^{\odot}$, Microsoft Flight Simulator $^{\odot}$ in VR, to name just a few), we argue that with XR still being a young discipline, there is a distinct lack of consistent terminologies and empirical studies. There is an emergent need to facilitate a discussion about technologies and designs tailored to environmental applications.

The remainder of the paper is structured as follows: We first review key concepts for embodied digital twins in Section 2. We then provide three examples of embodied digital twins for environmental applications in Section 3 discussing different scenarios and data. We conclude with a reflection on the concept of 
embodied digital twins and identify challenges in Section 4.

\section{EMBODIED DIGITAL TWINS - KEY CONCEPTS}

\subsection{Embodiment}

Understanding embodied digital twins requires to look first into the concept of embodiment and associated research. For centuries, mind and body were seen as being separate and the term mind-body dualism was coined (Crane and Patterson, 2000). Only in the last decades, researchers, inspired by advances in neurosciences, proposed scientific theories that offer an integrative view on mind and body and how the body and the simulation of experiences shape human cognition (Shapiro, 2019), giving older discussions such as metaphors and image schemata a scientific foundation (Lakoff and Johnson, 1980). The term used for this emerging perspective is embodied cognition (Shapiro, 2019). Embodied cognition acknowledges that the mind is not just an abstract information processor, rejecting the notion that cognition is amodal computation in a modular system (Barsalou, 2008); embodied cognition purports that the brain has evolved to control the human body (Clark, 1997) and that in return the body shapes our understanding of the physical world (Mandler, 1992). Acknowledging the body as an essential part of cognition elevates the role of local environments, too, as human perception and motor systems are interacting with the world through their senses (Barsalou, 2008).

There are philosophical nuances on the role of embodiment in cognition (Shapiro, 2019) but the key assumptions, which also explain the importance of embodiment for digital twins, can be summarized as follows (see (Wilson, 2002)): (1) Cognition is situated. (2) Cognition is time-pressured. (3) We off-load cognitive work to the environment. (4) The environment is part of the cognitive system. (5) Cognition is for action. (6) Off-line cognition is body based.

Understanding embodiment in light of these six key assumptions has facilitated its ever more important role in reforming approaches to learning, education, communication, and decision-making (Krishna and Schwarz, 2014). This paradigmatic change in understanding of how the mind works has provided a theoretical basis for understanding the importance of place and experiences that many disciplines intuitively adopt (see Section 1). Embodied cognition and its theoretical grounding help to explain why experiential learning and understanding are effective and efficient from a theoretical and human evolutionary perspective.

The emergence of embodied cognition also explains the excitement for immersive technologies becoming mainstream that has swept through numerous disciplines. Immersive technologies allow for truly embodied experiences of places and data and as such are a communication medium unlike traditional and other emerging technologies.

\subsection{Immersive Technologies}

We are using the term immersive technologies to characterize devices that have the capacity to physically immerse users by catering to their senses (Jerald, 2016). As the technology is still rather young and part of the canon of emerging technologies, terminological consistency is still not achieved and we find synonymous expressions such as XR, eXtended realities, or cross-realities. All of these terms refer to augmented, mixed,

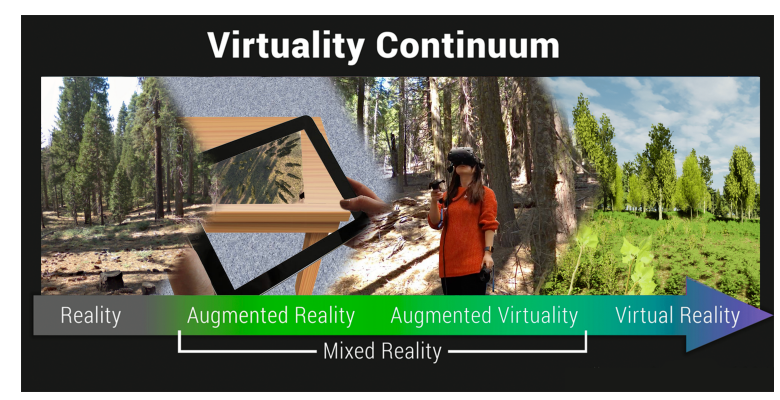

Figure 1 . The virtuality continuum first suggested by (Milgram and Kishino, 1994). It starts on the left with physical reality and arrives at a completely digital world on the right termed virtual reality. In between we find the realm of mixed reality that can

have several sub-concepts, here augmented reality and augmented virtuality. The difference is that in augmented reality digital content is superimposed onto the physical world, while augmented virtuality refers to replications of the physical world in a virtual reality setting (modified from (Klippel, 2020)).

or virtual reality and the technologies that allow users to experience digital content immersively. The term augmented reality is used to characterize technology that superimposes digital content onto the real, physical world (Wellner et al., 1993). Virtual reality refers to experiences that provide a consistent and complete virtual world to the users without access to the physical world (Blascovich and Bailenson, 2011). Mixed reality is situated between the two and not consistently defined. It can refer to an advanced version of augmented reality with content tailored to the physical world to provide seamless integration or replacement; we also find mixed reality as a summary term that encapsulates all forms of experiences where the virtual and the physical world are combined (Milgram and Kishino, 1994, Ohta and Tamura, 2014) (see Figure 1).

A word of caution: The term immersive has led to confusion in the academic and industrial world. The roots of this confusion can be traced back to the etymology of the term immersion. To immerse something (from Latin immersus) means to plunge it into a fluid. This original understanding of the term is the basis for the definition of immersive technologies as a user is physically, through various senses, "plunged" into a digital experience. We find that this definition is equivalent to "system characteristics" and is the dominant interpretation/usage in disciplines that are more technically-minded (Slater, 1999). We also find, however, that since the 17th century the term has been used figuratively referring to a cognitive state related to study, work, passion, or gaming. This latter understanding of immersion is widespread in the colloquial use of the term and prevalent in many disciplines, too (Liu et al., 2017). To avoid confusion, researchers have suggested to use the term presence to refer to the emotional and cognitive response of a user (Slater, 1999). They see presence as resulting from experiencing immersion and describe it as the feeling of "being there" (IJsselsteijn, 2003).

So what makes immersive technologies and resulting experiences different? The dichotomy that existed ever since the first graphical user interface, that is, the user on the one side of a display and the digital content on the other side, has disappeared. Users of immersive technologies are given high levels of agency that allow them to experience digital content from an egocentric perspective. The tracking capabilities of HMDs afford a user comparable agency to experiences in the real world. This agency is necessary to realize fully embodied experiences 


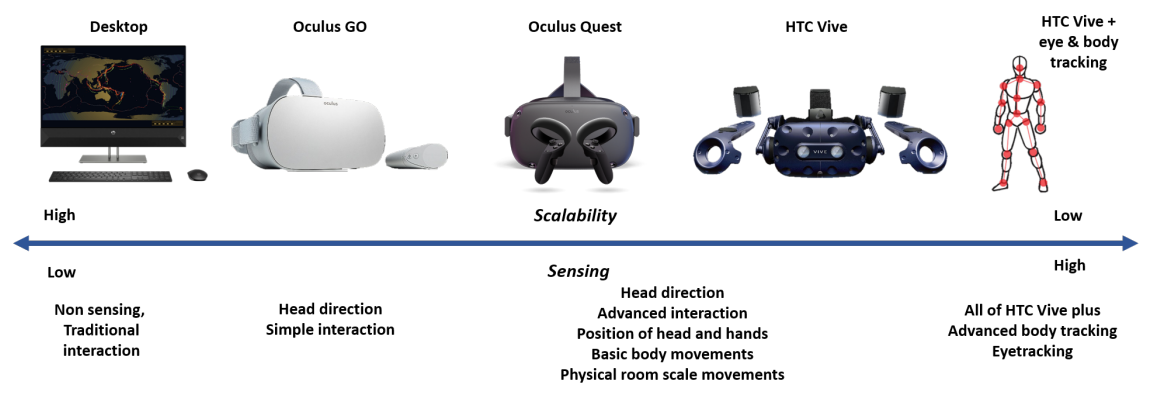

Figure 2. SENSATIUM, the SENsingScAlability Trade-off contInuUM (modified from (Klippel et al., 2020))

(see Section 2.1).

Immersive technologies have the potential to achieve the highest levels of embodiment through offering advanced sensing that maps every movement of the human body from the physical world into the digital realm. But, not all immersive technologies are created equal. In previous work we captured this aspect by defining SENSATIUM (Klippel et al., 2020, Klippel et al., 2019b), the sensing, scalability trade-off continuum (see Figure 2). As we move away from desktop environments, we first enter the realm of HMDs with three degrees of freedom, such as Oculus Go, that provide users with basic immersive experiences allowing for mapping head movements but no body movements. Devices with six degrees of freedom, such as Oculus Quest, provide mappings for head and body movements but are limited to information obtained from headsets and controllers. Full body sensing or at least a high-density sensor network allows for more nuanced mappings of body movements into the digital twin. While there are several studies on the effects of increased sensing on user experience and learning, results are limited and even contradictory (see Section 4). The effects of highest levels of embodiment on grounding environmental decision-making are largely unexplored.

\section{EXAMPLES OF ENVIRONMENTAL EMBODIED DIGITAL TWINS}

In this section, we will present and discuss three examples of embodied digital twins for environmental applications enabled through workflows for immersive experiences. We selected these examples to demonstrate the potential of embodied digital twins for (a) different kinds of data (remotely sensed and photogrammetric, simulated and procedurally modeled, and simulated based on real time and expert input); (b) place-based challenges such as remote and challenging to reach locations (inside of a volcano), invisible parts of the earth, and future environmental change.

\subsection{Iceland's Thrihnukar Volcano}

Excellent examples for the application of state-of-the art photogrammetry and remote sensing methods to create embodied digital twins are locations that are largely inaccessible. Some places are too remote or are largely unreachable such as the inside of a volcano. As part of our previous work to address this challenge, we developed an immersive workbench for the interpretation of geospatial datasets exemplarily realized for Iceland's Thrihnukar volcano (see (Zhao and Klippel, 2019a) for details). In the workflow to create an immersive VR version of the volcano, we combined satellite imagery data with a Digital Elevation Model (DEM) to create a basic reconstruction of the field site. We used Structure-from-Motion photogrammetry and terrestrial laser scanning to capture the interior structure of the volcano system. These datasets were georeferenced to provide high-resolution point clouds of the volcano's inside (Figure 3, left and middle) and coupled with an accurate textured terrain model depicting the location of the volcano system in the physical world. This visualization design benefits geoscientists because it promotes an understanding of spatial correlations between different data types, which is the key to making new discoveries in geoscience research.

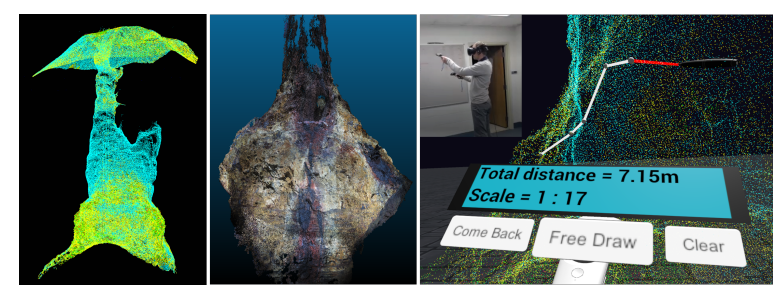

Figure 3. Thrihnukar experience in the immersive virtual environment (modified from (Zhao and Klippel, 2019a)). Left: terrestrial LiDAR data. Middle: Structure-from-Motion derived model preserving original point colors. Right: hand-based interaction with point clouds during length measurements.

The unique capability of immersive VR to view the 3D reconstruction of geospatial data naturally in its 3D format as an embodied experience makes a digital representation intuitive for exploring, understanding, and performing quantitative investigations (see also Figure 3). Our workbench offers tools for the direct manipulation of the georeferenced point clouds, including scaling, rotation, and translation, and a suite of precise tools for measuring the length, area, and volume of the magmaticvolcanic system. Particularly in the length-measuring activity, users are able to move their hand (a pen-like tool) to directly draw nodes and line segments on the point cloud (Figure 3 , right). The 6-DOF input device along with stable position tracking affords users the ability to "touch data at their fingertips" (Kreylos et al., 2006). Such natural and intuitive interaction mechanisms afforded through advanced embodiment are of prime importance for data exploration in the era of virtual reality. Thus, one unambiguous goal of creating an embodied digital twin for large and highly detailed datasets is to develop a visual analytic environment that allows users to use body-based sensory cues and embodied gestures to move through and interact with the data (Zhao and Klippel, 2019a).

\subsection{Critical Zone: Observatories and Gamification}

The critical zone (CZ) is the Earth's permeable near-surface layer from the top of the tree to the bottom of the groundwater (United States National Research Council, 2001). CZ observatories are an excellent example of transdisciplinary science 
and how massive instrumentation projects (White et al., 2015) provide environmental laboratories to study chemical, physical, and biological processes critical to humanities food, water, and energy supplies. Data collected at these sites is becoming available in real time through fiber optic cables and $5 \mathrm{G}$ networks. Being able to analyze such data in real-time and contextualized in actual environments is a tremendous opportunity for embodied digital twins as they allow researchers around the globe to participate in real-time, place-based collaboration.
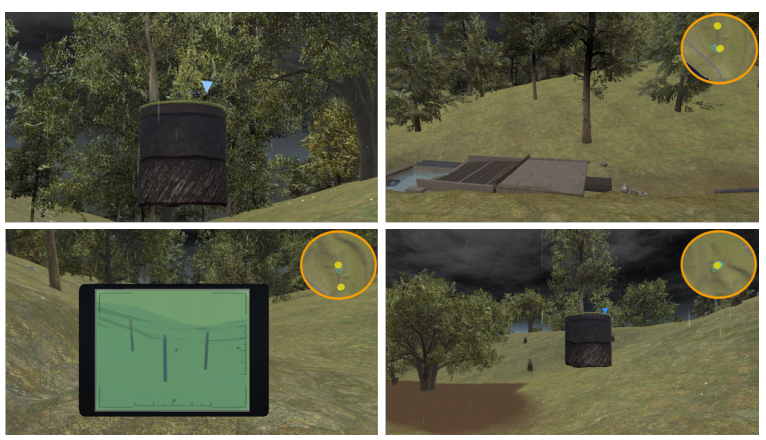

Figure 4. CZ Investigator: investigating the effect of natural and human process on the storage and flow of water.

Likewise, educational efforts can be grounded in real-world and real data examples. We developed CZ Investigator (Sajjadi et al., 2020), an immersive adventure serious game aimed at teaching the concept of the $\mathrm{CZ}$ to the general public (Figure 4). The player assumes the role of a journalist, tasked with writing an article about the effect of natural (e.g., rain) and human (e.g., deforestation) processes on the $\mathrm{CZ}$ in central PA. Players then need to go to the $\mathrm{CZ}$, investigate, gather evidence, and then report back before they decide on what to write. This narrative sets the basis for the four main educational objectives promoted by the game: 1) understanding what a Critical Zone is (based on a concrete experience rather than observing an abstract representation), 2) understanding the food-energy-water nexus in relation to the $\mathrm{CZ}$, and 3 ) exploring the effect of natural and human processes on the various components (lithosphere, hydrosphere, biosphere, and atmosphere) of a CZ (Figure 4).

The natural environment of the game can be described as a "model of reality" (Harteveld, 2011), modelled based on a DEM created from the actual CZ site. High-resolution Lidar data from 2010 with an average of 10 points $/ \mathrm{m}^{2}$ with $2-4 \mathrm{~cm}$ vertical accuracy were used to create the DEM in ArcGIS, and was then converted to RAW image, and consequently a 3D terrain in the Unity3D game engine. The natural environment was then procedurally populated with a variety of vegetation and tree species based on a 2016 survey of the trees and elements found in the central PA CZ, with a reasonable level of accuracy. Such a model of reality not only facilitates the replication of a real environment (with a reasonable accuracy), but also enables us to perform live manipulation of environmental variables (e.g., water storage and flow, soil moisture, etc.) based on real-time data captured by sensors and transmitted from the actual site.

\subsection{Visualizing Forest Futures}

As part of a project that aims at communicating the effects of climate change to decision makers and the general public, we created a data-driven immersive VR forest landscape under different climate scenarios. The result is a comprehensive forest ecosystem, including topography, species composition and density, coarse woody debris, and understory conditions which can be viewed, experienced, and interacted with in immersive VR (Huang et al., 2020).

The project demonstrates how linking ecological modeling, procedural modeling, and VR can provide an immersive, visceral experience of a future forest. We mapped current tree species composition in northern Wisconsin using the Forest Inventory and Analysis (FIA) data and then forecast forest change 50 years into the future under two climate scenarios, using a spatially-explicit, mechanistic simulation model (LANDIS-II) (Scheller et al., 2007). Procedural modeling with Computer Generated Architecture rules was used to create the tree overstory based on the model output. Tree saplings, ferns, bushes, ground plants, grasses, wildflowers and snags that are commonly found in the region were added to the forest. With the full environment generated, we added environment interactions, climate scenarios exploration and information retrieval in the Unreal Engine (Huang et al., 2020).

We validated our procedural modeling approach by applying it to FIA plots data without the added layer of climate change projection and simulation. The comparison between the immersive VR representation and a field photograph is shown in Figure 5.

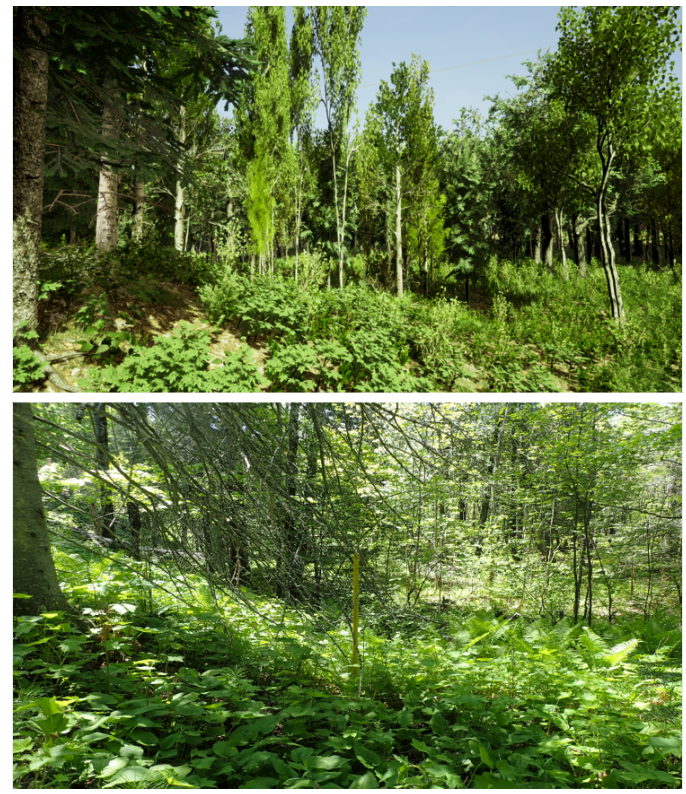

Figure 5. Top: a forest modeled based on FIA dataset without the simulation; bottom: photograph taken at the same FIA plot.

\section{DISCUSSION, CHALLENGES, OPPORTUNITIES}

As part of the Introduction and Section 3, we have argued that embodied digital twins are particularly important for environmental applications due to the place-based nature of associated disciplines. Furthermore, we argue that immersive technologies provide a medium that allows for designing cognitively ergonomic representations as they transcend the dichotomy of users separated from representations allowing the user to step into the data, gain agency, and access data through an embodied experience. We find in Wilson's six views on embodiment (Wilson, 2002) (see again Section 2.1) the theoretical grounding for why embodying digital twins is such an important development and that embodied cognition can play a central role in advancing 
the theory of immersive visualizations for environmental applications. In her assessment, arguments 1, 3, 4, and 6 are almost self-explanatory: Embodied digital twins are situating the user, allow for off-loading cognitive tasks, make the environment part of the cognitive system, and create body-based experiences that lend themselves to a lasting understanding and memory. We can also envision that aspects of time-pressure as understood by Wilson are part of embodied digital twins in that situated cognition takes part in real time and that situating the user, hence, mimics that experience. Finally, understanding that cognition is for action is a superb example of advantages of digital twins as pointed out by Glenberg (Glenberg, 1997), cited in (Wilson, 2002)): "[cognition] evolved in service of perception and action in a three-dimensional environment." These arguments have inspired research on immersive learning environments (Liu et al., 2017) as well as on immersive analytics (Chandler et al., 2015, Dwyer et al., 2018), and we are only at the beginning to fully comprehend and theorize the potential of immersive technologies and embodied digital twins.

There are many open questions that a growing and diverse research community is addressing. We will briefly discuss four of them here.

Does embodiment through immersion really help? Across the research and educational community, there is a rapidly growing number of studies addressing the potential of immersive research, learning, and decision-making compared to traditional media such as desktop applications. One of the best established benefits of virtual environments are their positive effects on training and transfer of knowledge (Liu et al., 2017). This is critical for establishing embodied digital twins for environmental applications as we often expect knowledge acquired in the virtual world to be used in real-world settings. Studies also have shown that virtual field trips are a valid substitute of actual field trips, at least for those that are within a 90 minute time frame (Klippel et al., 2019a), and that experiences can be enhanced by using pseudo-aerial imagery integrated into the virtual field trips (Zhao and Klippel, 2019b). Additionally, studies show that higher levels of immersion and interaction fidelity lead to improved results both in terms of performance measures and self-efficacy of participants (Krokos et al., 2018, Klingenberg et al., 2020). Across a range of academic disciplines, findings indicate that immersive VR environments are promoting motivational outcomes and offer superior learning experiences compared to traditional desktops and even real world experiences (Makransky et al., 2020). However, assessment of learning performance and analysis of cognitive factors have also yielded mixed results. We do find that immersive VR lead to inconclusive or no differences (Bursztyn et al., 2017, Jensen and Konradsen, 2018); and, in some cases can even show negative effects (Parong and Mayer, 2018).

Summarizing these findings shows the need for research frameworks that go beyond individual studies and that systematically address open research questions. It is clear that immersion and resulting embodiment alone are not sufficient to consistently improve user experience and performance. What is necessary is a concerted effort to understand the interplay between immersive technologies and design choices that realize how embodied digital twins are experienced. We briefly discussed presence in Section 2 and have previously summarized the behavioral responses of a user (Klippel, 2020). While there is ongoing debate, there are four main concepts that can be identified: Spatial presence, which is characterized as the sense of being there (IJsselsteijn, 2003); co-presense or social presence, which captures the connection that a user may develop toward other agents, whether they are artificial or human (Biocca et al., 2003); flow is used to characterize how well the mechanics, challenges, and rules of an experiences engage a user (Csikszentmihalyi, 1991, Shernoff et al., 2003); and finally the narrative or sequence, which stresses how deeply a user is invested in the story or experience anticipating the sequence of events (Qin et al., 2009). In addition to these four concepts that broadly characterize experiences associated with embodied digital twins, we find a rapidly growing number of studies that develop and use a variety of assessment instruments to establish the effects of immersive experiences on users in a more nuanced way. We already mentioned performance and self-efficacy above. Additionally we find studies that focus on media effects such as awe or novelty (Chirico et al., 2018), or develop sophisticated measures such as ripple effects (Sajjadi et al., 2020). The bottom line is that the relative youth of embodiment enabled through technology so far has not allowed for establishing deep theoretical foundations, despite several decades of research.

Uncertainty. One of the biggest challenges of embodied digital twins for environmental applications is the dichotomy of uncertainty and realism, that might be coined the uncertaintyrealism-dualism. Uncertainty has always played a key role in the geospatial sciences (Goodchild, 2020), and the more complex the system or the farther we move into the past or future, chances are that uncertainty will increase. The example of visualizing forest futures (Section 3.3) is an excellent example as we have the traditional uncertainty sources and additionally decisions that are required to turn abstract data into a specific three-dimensional model, out of an infinite number of possible realizations. Given that uncertainty is challenging for the human mind to start with, presenting users with a concrete example for what really is a set of possible solutions might have unknown side effects. Not every embodied digital twin will have these issues but it will require careful considerations and empirical research to find the right balance or the right means to communicate uncertainty through concrete experiences.

Scale. Scale has long been recognized as a critical component in environmental sciences (Buizer et al., 2011, Gibson et al., 2000). The understanding of any phenomenon on earth, cognitively speaking, is a scale-dependent process such that the way a phenomenon is described and explained strongly depends on how it is being observed and measured. Many environmental problems, such as climate change, pollution, and floods are characterized by complex inter-dependencies across multiple scales (Termeer and Dewulf, 2014). Without dealing appropriately with scale, these problems are often difficult to understand and sometimes misleading.

It is obvious that integrating the concept of embodied digital twins into scale-sensitive environmental issues is promising and even necessary as a means to translate information across local and global scales. Embodied digital twins challenge the traditional notions of scale because of their dual nature being at the same time representations of environments, and environments themselves (Montello et al., 2004). The use of embodiment further allows users to switch fluidly between different levels of representations through the natural interactions with virtual devices and objects.

Dede (2009) has made an important observation highlighting the value of embodied digital twins to unravel the multilevel nature of environmental issues. He suggests that immersive 
experiences can enhance the understanding of a complex phenomenon by shifting between inside (e.g., egocentric) and outside (e.g., bird's-eye) perspectives. An inside perspective can induce presence and therefore should foster users' intrinsic motivation to learn; an outside perspective supports more abstract, symbolic insights when the environment is contextualized holistically (Dede, 2009). An embodied digital twin that allows for alternating between these perspectives has the potential to combine these benefits.

Digital twins are essentially a spatial concept and sometimes can be conceived of as "soft-where" in view of their endless possibilities for ingesting real-world environmental data and information (Foreman and Korallo, 2014). Since a virtual world can represent any scale of nature, ranging from an atom to the earth, the perceived size of a representation is apparently independent of one's real body. Given that space is experienced in a scale-dependent way (Montello, 1993), the extent to which such novel scale manipulations affect human environmental perception remains a largely open question.

Behavior monitoring and adaptivity. Extensive argumentation can be found in the literature for adapting virtual environments to the needs and preferences of users (e.g., (Lopes and Bidarra, 2011, Sajjadi et al., 2014)). One of the main advantages that virtual environments offer is the implicit (stealth) assessment (Shute, 2011) of user states based on their behavior. This information can help designers and researchers in implementing intelligent mechanisms for profiling users and adapting the environment to cater to their individual needs and abilities.

While a plethora of "user aspects" (e.g., performance, preferences, knowledge level, attention, physiological signals) have been studied in relation to adaptation, immersive VR offers new forms of user data that can be used in this domain. In an embodied digital twin, users actively need to move around, similar to what they do in the real-world, and interact with digital objects by performing gestures that are natural to them. As such, the users are constantly producing big data pertaining to their movements with six degrees of freedom. When combined with conventional user aspects, this information can be potentially used to predict the attentiveness, competencies, misconceptions, frustration, engagement, motivation, and more meaningful states of users in real-time (e.g., (Winkler-Schwartz et al., 2019, Alcañiz Raya et al., 2020)). This area of monitoring, classifying, modeling, and predicting user characteristics and behavior is one of the main areas in which embodied digital twins can benefit from Artificial Intelligence and Machine Learning methods (other areas are reconstruction, content creation, and analytics support (Lock et al., 2019)). Such meaningful predictions can then be used to tailor a virtual experience to an individual level. This opens the door for numerous interesting research questions from the design of intelligent and adaptive embodied digital twins, to evaluating the effectiveness of adaptation in educational, research, communication, and decision-making domains.

\section{CONCLUSIONS}

In this paper we started a discussion and potential theoretical grounding of embodied digital twins. We pointed out that there is a disconnect between the focus on place in the environmental sciences and emerging theories on embodied cognition in the cognitive sciences. Actively discussing embodied cognition will provide a grounding of a science of embodied digital twins for environmental applications. However, examining the literature, we find that embodiment, achieved through immersive technologies, alone is not sufficient by itself. While immersive technologies, especially at the upper end of SENSATIUM, provide the most sophisticated and natural interface for embodied experiences and embodied digital twins, the design of the experiences has to be placed in the foreground. This has been the case for a long time in the gaming community (Harteveld, 2011) as well as the immersive learning research community (Liu et al., 2017) and it is pivotal for environmental sciences to integrate this research into their theories about place and to think about suitable research frameworks.

We also identified challenges and opportunities for embodied digital twins. We exemplary discussed three topics that are central to geospatial sciences as well as immersive learning and research approaches: Uncertainty, scale, and behavioral monitoring and adaptivity. We believe that these topics, in combination with the theoretical grounding achieved through combining place and embodiment, have the potential to advance the science of embodied digital twins and we hope that this vision paper provides a springboard for fruitful discussions.

\section{REFERENCES}

Alcañiz Raya, M., Marín-Morales, J., Minissi, M. E., Teruel Garcia, G., Abad, L., Chicchi Giglioli, I. A., 2020. Machine learning and virtual reality on body movements' behaviors to classify children with autism spectrum disorder. Journal of clinical medicine, 9(5), 1260 .

Bailenson, J., 2018. Experience on demand: What virtual reality is, how it works, and what it can do. W. W. Norton \& Company, New York, NY.

Barab, S., Dede, C., 2007. Games and immersive participatory simulations for science education: An emerging type of curricula. Journal of Science Education and Technology, 16(1), 13.

Barsalou, L. W., 2008. Grounded cognition. Annual Review of Psychology, 59, 617-645.

Biocca, F., Harms, C., Burgoon, J. K., 2003. Toward a More Robust Theory and Measure of Social Presence: Review and Suggested Criteria. Presence: Teleoperators and Virtual Environments, 12(5), 456-480.

Blascovich, J., Bailenson, J., 2011. Infinite reality: Avatars, eternal life, new worlds, and the dawn of the virtual revolution. 1 st ed. edn, William Morrow, New York.

Buizer, M., Arts, B., Kok, K., 2011. Governance, Scale and the Environment: The Importance of Recognizing Knowledge Claims in Transdisciplinary Arenas. Ecology and Society, 16(1). http://www.ecologyandsociety.org/vol16/iss1/art21/.

Bursztyn, N., Walker, A., Shelton, B., Pederson, J., 2017. Assessment of student learning using augmented reality Grand Canyon field trips for mobile smart devices. Geosphere, 13(2), 260-268.

Chandler, T., Cordeil, M., Czauderna, T., Dwyer, T., Glowacki, J., Goncu, C., Klapperstueck, M., Klein, K., Marriott, K., Schreiber, F., Wilson, E., 2015. Immersive analytics. 2015 Big Data Visual Analytics (BDVA), 1-8. 
Chirico, A., Ferrise, F., Cordella, L., Gaggioli, A., 2018. Designing Awe in Virtual Reality: An Experimental Study. Frontiers in psychology, 8. https://www.frontiersin.org/articles/10.3389/fpsyg.2017.02351/full.

Clark, A., 1997. Being there: Putting brain, body, and world tegether again. MIT Press, Cambridge, MA.

Coltekin, A., Lochhead, I., Madden, M., Christophe, S., Devaux, A., Pettit, C., Lock, O., Shukla, S., Herman, L., Stachoň, Z., Kubíček, P., Snopková, D., Bernardes, S., Hedley, N., 2020. Extended Reality in Spatial Sciences: A Review of Research Challenges and Future Directions. ISPRS International Journal of Geo-Information, 9(7).

Cox, A., Hart, R. B., 1986. Plate tectonics: How it works / Allen Cox, Robert Brian Hart. Blackwell Scientific, Palo Alto, Calif and Oxford.

Crane, T., Patterson, S., 2000. History of the mind-body problem. London studies in the history of philosophy, 3, Routledge, London.

Csikszentmihalyi, M., 1991. Flow: The psychology of optimal experience : steps toward enhancing the quality of life. Harper Collins Publishers, New York, NY.

Dede, C., 2009. Immersive interfaces for engagement and learning. Science (New York, N.Y.), 323(5910), 66-69.

Dolphin, G., Dutchak, A., Karchewski, B., Cooper, J., 2019. Virtual field experiences in introductory geology: Addressing a capacity problem, but finding a pedagogical one. Journal of Geoscience Education, 67(2), 114-130.

Dwyer, T., Marriott, K., Isenberg, T., Klein, K., Riche, N., Schreiber, F., Stuerzlinger, W., Thomas, B. H., 2018. Immersive analytics: An introduction. K. Marriott, F. Schreiber, T. Dwyer, K. Klein, N. Riche, T. Itoh, W. Stuerzlinger, B. H. Thomas (eds), Immersive analytics, 11190, Springer, Cham, Switzerland, 1-23.

Foreman, N., Korallo, L., 2014. Past and future applications of 3-D (virtual reality) technology. Scientific and Technical Journal of Information Technologies, Mechanics and Optics, 6(94), 1-8.

Gibson, C. C., Ostrom, E., Ahn, T. K., 2000. The concept of scale and the human dimensions of global change: A survey. Ecological Economics, 32(2), 217-239.

Glenberg, A. M., 1997. What is memory for? Behavioral and Brain Sciences, 20(1), 1-55.

Goodchild, M. F., 2020. How well do we really know the world? Uncertainty in GIScience. Journal of Spatial Information Science.

Grieves, M., Vickers, J., 2017. Digital twin: Mitigating unpredictable, undesirable emergent behavior in complex systems. F.-J. Kahlen, S. Flumerfelt, A. Alves (eds), Transdisciplinary perspectives on complex systems, Springer, Cham, Switzerland, 85-113.

Harteveld, C., 2011. Triadic game design: Balancing reality, meaning and play. Springer Science \& Business Media.
Huang, J., Lucash, M. S., Scheller, R. M., Klippel, A., 2020. Walking through the forests of the future: using data-driven virtual reality to visualize forests under climate change. International Journal of Geographical Information Science, 1-24.

IJsselsteijn, W. A., 2003. Presence in the past: What can we learn from media history? W. A. IJsselsteijn, G. Riva, F. Davide (eds), Being there, Emerging communication. Studies in new technologies and practices in communication, IOS, Amsterdam and Oxford, 17-40.

Jensen, L., Konradsen, F., 2018. A review of the use of virtual reality head-mounted displays in education and training. Education and Information Technologies, 23(4), 1515-1529.

Jerald, J., 2016. The VR Book: Human-centered design for virtual reality. ACM Books, 8, first edition edn, Association for Computing Machinery and Morgan \& Claypool Publishers, New York, NY and San Rafael.

Klingenberg, S., Jørgensen, M. L. M., Dandanell, G., Skriver, K., Mottelson, A., Makransky, G., 2020. Investigating the effect of teaching as a generative learning strategy when learning through desktop and immersive VR: A media and methods experiment. British Journal of Educational Technology, online first.

Klippel, A., 2020. From spatial to platial - the role and future of immersive technologies in the spatial sciences. Journal of Spatial Information Science, 33-45.

Klippel, A., Zhao, J., Jackson, K. L., LaFemina, P., Stubbs, C., Oprean, D., Wallgrün, J. O., Blair, J., 2019a. Transforming earth science education through immersive experiences - delivering on a long held promise. Journal of Educational Computing Research, 57(7), 1745-1771.

Klippel, A., Zhao, J., Oprean, D., Wallgrün, J. O., Chang, J. S.-K., Wallgrun, J. O., Chang, J. S.-K., 2019b. Research framework for immersive virtual field trips. IEEE Conference on Virtual Reality and 3D User Interfaces. KELVAR: The Fourth IEEE VR Workshop on K-12+ Embodied Learning through Virtual and Augmented Reality., IEEE, 1612-1617.

Klippel, A., Zhao, J., Sajjadi, P., Wallgrün, J. O., Bagher, M. M., Oprean, D., 2020. Immersive place-based learning - an extended research framework. 2020 IEEE Conference on Virtual Reality and 3D User Interfaces Abstracts and Workshops (VRW), IEEE, Piscataway, NJ, 449-454.

Kreylos, O., Staadt, O. G., Sumner, D. Y., Bawden, G., Bernardin, T., Billen, M. I., Cowgill, E. S., Gold, R. D., Hamann, B., Jadamec, M., Kellogg, L. H., 2006. Enabling scientific workflows in virtual reality. S. N. Spencer (ed.), Proceedings VRCIA 2006, Association for Computing Machinery Inc, New York, $155-162$.

Krishna, A., Schwarz, N., 2014. Sensory marketing, embodiment, and grounded cognition: A review and introduction. Journal of Consumer Psychology, 24(2), 159-168.

Krokos, E., Plaisant, C., Varshney, A., 2018. Virtual memory palaces: Immersion aids recall. Virtual Reality, 2(1-2), 89.

Lakoff, G., Johnson, M., 1980. Metaphors we live by. University of Chicago Press, Chicago. 
Lin, H., Batty, M., Jørgensen, S. E., Fu, B., Konecny, M., Voinov, A., Torrens, P., Lu, G., Zhu, A.-X., Wilson, J. P., Gong, J., Kolditz, O., Bandrova, T., Chen, M., 2015. Virtual environments begin to embrace process-based geographic analysis. Transactions in GIS, 19(4), 493-498.

Liu, D., Dede, C., Huang, R., Richards, J. (eds), 2017. Virtual, Augmented, and Mixed Realities in Education. Smart Computing and Intelligence, Springer, Singapore.

Lock, O., Bain, M., Pettit, C., 2019. Urbanai - developing machine learning approaches and interfaces to support the planning and delivery of transport and housing in sydney.

Lopes, R., Bidarra, R., 2011. Adaptivity challenges in games and simulations: a survey. IEEE Transactions on Computational Intelligence and AI in Games, 3(2), 85-99.

Makransky, G., Petersen, G. B., Klingenberg, S., 2020. Can an immersive virtual reality simulation increase students' interest and career aspirations in science? British Journal of Educational Technology.

Mandler, J. M., 1992. How to build a baby II. Conceptual primitives. Psychological Review, 99(4), 587-604.

McComas, W. F. (ed.), 2014. The Language of Science Education: An Expanded Glossary of Key Terms and Concepts in Science Teaching and Learning. SensePublishers and Imprint: SensePublishers, Rotterdam.

Milgram, P., Kishino, F., 1994. A taxonomy of mixed reality visual displays. IEICE TRANSACTIONS on Information and Systems, E77-D(12), 1321-1329.

Montello, D. R., 1993. Scale and multiple psychologies of space. A. U. Frank, I. Campari (eds), Spatial information theory, Lecture Notes in Computer Science, 716, Springer-Verlag, Berlin and New York, 312-321.

Montello, D. R., Hegarty, M., Richardson, A. E., Waller, D., 2004. Spatial memory of real environments, virtual environments, and maps. G. L. Allen (ed.), Human spatial memory, Lawrence Erlbaum, Mahwah, N.J. and London, 251-285.

Ohta, Y., Tamura, H., 2014. Mixed Reality: Merging Real and Virtual Worlds. 1 edn, Springer Publishing Company, Incorporated.

Parong, J., Mayer, R. E., 2018. Learning science in immersive virtual reality. Journal of Educational Psychology.

Powers, A. L., 2004. An evaluation of four place-based education programs. The Journal of Environmental Education, 35(4), $17-32$.

Qin, H., Patrick Rau, P.-L., Salvendy, G., 2009. Measuring player immersion in the computer game narrative. International Journal of Human-Computer Interaction, 25(2), 107-133.

Sajjadi, P., Bagher, M. M., Cui, Z., Myrick, J. G., Swim, J. K., White, T. S., Klippel, A., 2020. Design of a serious game to inform the public about the critical zone. 2020 IEEE 8th International Conference on Serious Games and Applications for Health (SeGAH), IEEE, 1-8.

Sajjadi, P., Van Broeckhoven, F., De Troyer, O., 2014. Dynamically adaptive educational games: A new perspective. International Conference on Serious Games, Springer, 71-76.
Scheller, R. M., Domingo, J. B., Sturtevant, B. R., Williams, J. S., Rudy, A., Gustafson, E. J., Mladenoff, D. J., 2007. Design, development, and application of LANDIS-II, a spatial landscape simulation model with flexible temporal and spatial resolution. Ecological Modelling, 201(3-4), 409-419.

Semken, S., Ward, E. G., Moosavi, S., Chinn, P. W. U., 2018. Place-based education in geoscience: Theory, research, practice, and assessment: Theory, Research, Practice, and Assessment. Journal of Geoscience Education, 65(4), 542-562.

Shapiro, L. A., 2019. Embodied cognition. New problems of philosophy, second edition edn, Routledge, New York.

Shernoff, D. J., Csikszentmihalyi, M., Shneider, B., Shernoff, E. S., 2003. Student engagement in high school classrooms from the perspective of flow theory. School Psychology Quarterly, 18(2), 158-176.

Shute, V. J., 2011. Stealth assessment in computer-based games to support learning. Computer games and instruction, 55(2), 503-524.

Slater, M., 1999. Measuring Presence: A Response to the Witmer and Singer Presence Questionnaire. Presence: Teleoperators and Virtual Environments, 8(5), 560-565.

Tao, F., Zhang, H., Liu, A., Nee, A. Y. C., 2019. Digital twin in industry: State-of-the-art. IEEE Transactions on Industrial Informatics, 15(4), 2405-2415.

Termeer, C., Dewulf, A., 2014. Scale-sensitivity as a governance capability: Observing, acting and enabling. F. Padt, P. Opdam, N. Polman, C. Termeer (eds), Scale-sensitive governance of the environment, John Wiley \& Sons Inc, Chichester, West Sussex and Hoboken, NJ.

United States National Research Council, 2001. Basic Research Opportunities in Earth Sciences.

Wellner, P., Mackay, W., Gold, R., 1993. Back to the real world. Communications of the ACM, 36(7), 24-27.

White, T., Brantley, S., Banwart, S., Chorover, J., Dietrich, W., Derry, L., Lohse, K., Anderson, S., Aufdendkampe, A., Bales, R., Kumar, P., Richter, D., McDowell, B., 2015. Chapter 2: The role of critical zone observatories in critical zone science. Developments in Earth Surface Processes, 19, 15-78.

Wilson, M., 2002. Six views of embodied cognition. Psychonomic Bulletin and Review, 9(4), 625-636.

Winkler-Schwartz, A., Yilmaz, R., Mirchi, N., Bissonnette, V., Ledwos, N., Siyar, S., Azarnoush, H., Karlik, B., Del Maestro, R., 2019. Machine learning identification of surgical and operative factors associated with surgical expertise in virtual reality simulation. JAMA network open, 2(8), e198363-e198363.

Zhao, J., Klippel, A., 2019a. Scale - unexplored opportunities for immersive technologies in place-based learning. R. Teather, Y. Itoh, J. Gabbard (eds), Proceedings of the 26th IEEE Conference on Virtual Reality and 3D User Interfaces, IEEE, Piscataway, NJ, 155-162.

Zhao, J., Klippel, A., 2019b. Scale - unexplored opportunities for immersive technologies in place-based learning. 2019 IEEE Conference on Virtual Reality and $3 D$ User Interfaces (VR), Osaka, Japan., IEEE, 155-162. 